\title{
www.czasopisma.pan.pl \\ Destruction Mechanism of ZnAl4 as Cast Alloy Subjected to Cavitational Erosion Using Different Laboratory Stands
}

\author{
R. Jasionowski ${ }^{a} *$, W. Polkowski ${ }^{\mathrm{b}}$, D. Zasada ${ }^{\mathrm{b}}$ \\ ${ }^{a}$ Institute of Basic Technical Sciences, Maritime University of Szczecin, Szczecin, Poland \\ ${ }^{b}$ Faculty of Advanced Technology and Chemistry, Military University of Technology, Warszawa, Poland \\ *Corresponding author. E-mail address: r.jasionowski@am.szczecin.pl
}

Received 26.05.2015; accepted in revised form 17.07.2015

\begin{abstract}
The main reason of a cavitational destruction is the mechanical action of cavitation pulses onto the material's surface. The course of cavitation destruction process is very complex and depends on the physicochemical and structural features of a material. A resistance to cavitation destruction of the material increases with the increase of its mechanical strength, fatigue resistance as well as hardness. Nevertheless, the effect of structural features on the material's cavitational resistance has been not fully clarified. In the present paper, the cavitation destruction of $\mathrm{ZnAl} 4$ as cast alloy was investigated on three laboratory stands: vibration, jet-impact and flow stands. The destruction mechanism of ZnAl4 as cast alloy subjected to cavitational erosion using various laboratory stands is shown in the present paper.
\end{abstract}

Keywords: Cavitation, Cavitation wear, ZnAl4 alloy

\section{Introduction}

Sudden pressure changes in a flowing liquid are mainly responsible for the cavitational destruction. Pulse pressure variations in the liquid below the critical pressure (that is closed to the evaporating pressure of the liquid at a given temperature) leads to a formation of gas and steam bubbles that subsequently implode in a higher pressure zone [1-6]. The cavitation erosion results in the destruction of a material starting from the strengthening of surface layer and changes in the mechanical properties. After that, the strengthened surface layer of the material becomes brittle, which facilitates a nucleation and a formation of cracks, a detachment of single grains or agglomerates of grains or the formation of craters. The course of cavitation destruction process is very complex and depends on the physicochemical and structural features of a material. A lot of research has been devoted to the study on a correlation between materials properties such as a hardness, a yield strength and a tensile strength, elastic modulus and a material's cavitation behavior [7-10].

In this paper, the mechanism of cavitational destruction of ZnAl4 as cast alloy is shown.

\section{Material and methods}

The research was carried out on ZnAl4 alloy fabricated by a melting and casting of pure elements (Zn $99.995 \%$ and Al $99.7 \%$ ) in PIT10 induction furnace. 
As cast samples were subsequently analyzed on three various stands for the cavitational erosion evaluation namely, vibration, jet impact and flow stands. In the case of the vibration stand the main induced phenomenon is the acoustic cavitation. This type of destruction is usually observed in cylinder coats of liquid-cooled engines; in echo radars near the membranes and in the strongly loaded sliding bearings working at high sliding speeds. A view of the vibration stand used in the present work is shown in Fig. 1.

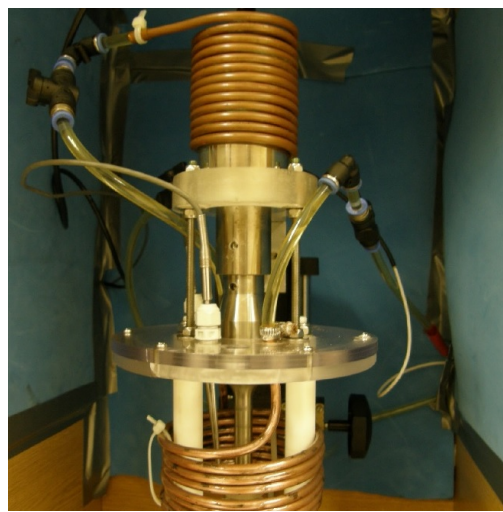

Fig. 1. A view of the vibration stand used in the present work

The analysis was conducted on three MgAl2Si alloy samples having cylindrical shape with $12.3 \pm 0.1 \mathrm{~mm}$ diameter and $5 \pm 0.1$ $\mathrm{mm}$ height. The samples were mounted in a holder placed opposite to the end of the oscillation resonator at a distance of $2.5 \mathrm{~mm}$. The relative amplitude was equal to $80 \%$ of the nominal amplitude of $124 \mu \mathrm{m}$. Demineralized water was the working liquid, maintained at a temperature of $21 \div 22{ }^{\circ} \mathrm{C}$. Following exposition times were applied: 1, 5, 15, 30, 60, 90 and 120 minutes.

Samples that are analyzed on the jet impact stand exhibit very low mass losses induced by a cavitation cloud. The water beam flowing through a nozzle hits a sample's surface leading to an implosion of cavitation bubbles, and thus a cavitation erosion.

A macroview of the jest impact stand is show in Fig. 2.

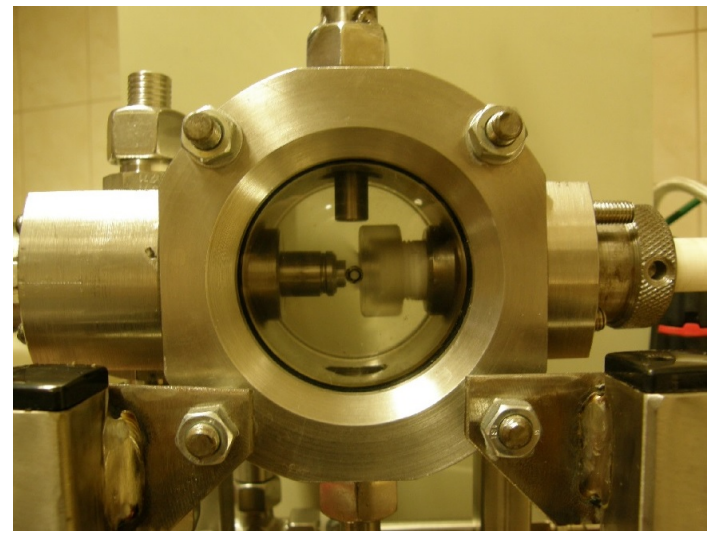

Fig. 2. A macroview of the jet impact stand used in the present work
The analysis was conducted on three MgAl2Si alloy samples having cylindrical shape with $12.3 \pm 0.1 \mathrm{~mm}$ diameter and $5 \pm 0.1 \mathrm{~mm}$ height. The samples were mounted in a holder placed opposite to the nozzle with $0.4 \mathrm{~mm}$ diameter at a distance of $5 \mathrm{~mm}$. The liquid pressure was set to 125 bar. In the chamber was pumped with a pressure of 5 bar, while a flow rate was $5 \mathrm{dm}^{3} / \mathrm{min}$. Demineralized water was the working liquid, maintained at a temperature of $18 \div 20{ }^{\circ} \mathrm{C}$. Following exposition times were applied: $15,30,60$ and 120 minutes.

Finally, the flow stand was also used upon the research (Fig. 3).

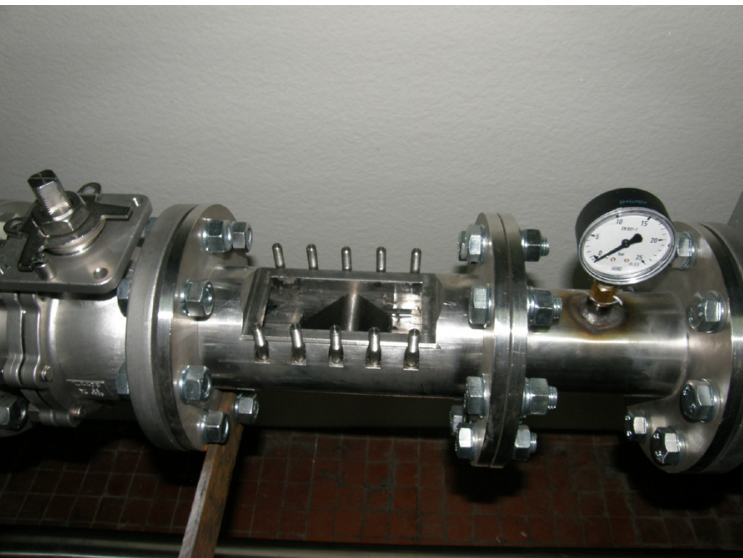

Fig. 3. A view of the cavitation tunnel chamber of the flow stand used in the present work

In this device the cavitation phenomena is induced by a crosssectional profile of the flow beam causes a pressure drop, and a local increase of liquid velocity. A cavitation tunnel chamber has dimensions of $55 \times 55 \mathrm{~mm}$ with a wedge shaped cavitation inductor. PML2 pump powered by a $15 \mathrm{~kW}$ engine produces a working fluid pressure of approximately $5000 \mathrm{hPa}$. The working fluid was tap water with a temperature in the range of 20 to $24{ }^{\circ} \mathrm{C}$. The specimens had a parallelepiped shape with dimensions of $30 \times 55 \times 6 \mathrm{~mm}$. Following exposition times were applied: 15, 60, 120, 180, 240, and 300 minutes.

All samples of the ZnAl4 alloy after the examination of cavitation erosion were removed from the test equipment degreased in an ultrasonic bath for a period of 3 minutes at $30^{\circ} \mathrm{C}$, dried in the oven for 5 minutes at $120^{\circ} \mathrm{C}$ and then weighted.

\section{Results and discussion}

The structure of ZnAl4 as cast alloy is characterized by $\eta$ solid solution dendrites and $\eta+\alpha$ (Fig. 4). 


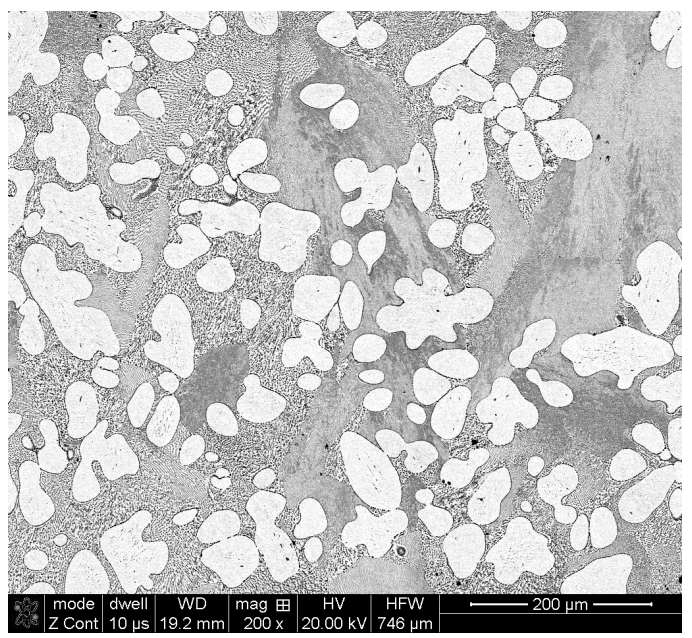

Fig. 4. Microstructure of ZnAl4 as cast alloy

The cavitation destruction the ZnAl4 alloy tested on the vibration stand starts with a depletion of $\eta+\alpha$ eutectic in grain and a)

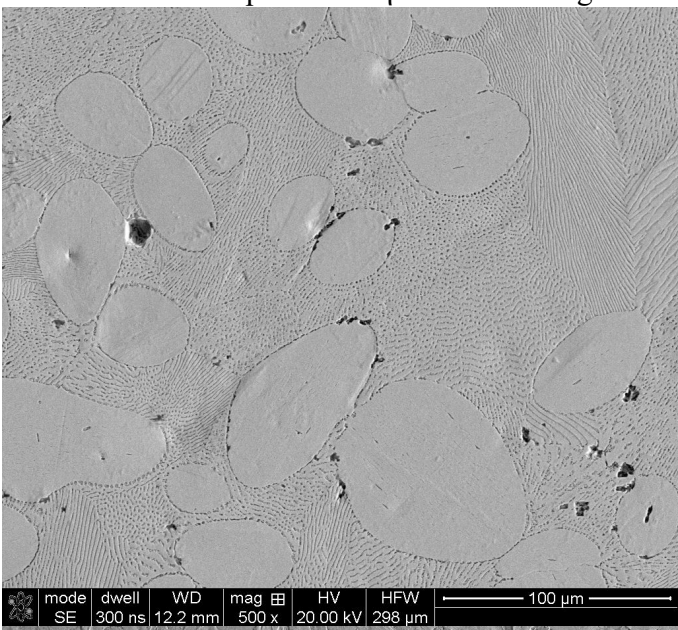

c)

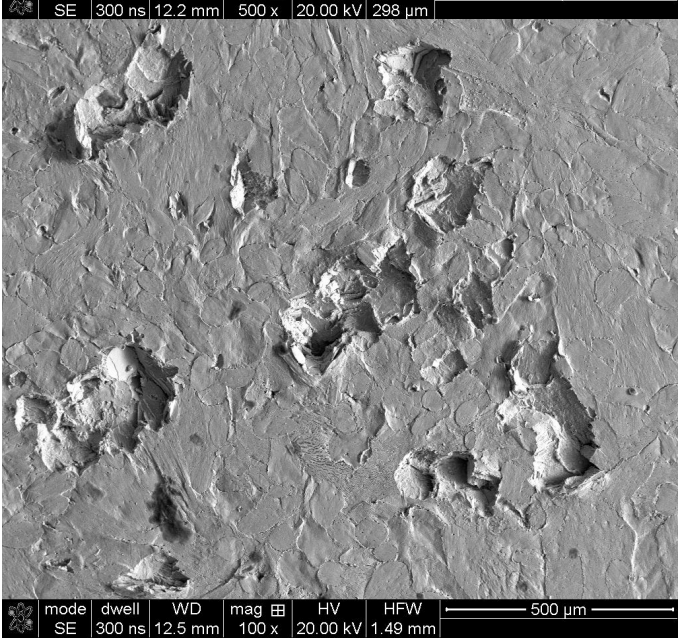

phase boundaries zones (Fig. 5a). Additionally, some traces of plastic deformation are also visible on the sample's surface (Fig. $5 b)$. Due to the erosion of $\eta+\alpha$ eutectic and a fatigue damage, whole grains of the $\eta$ solid solution or their large parts were detached (Fig. 5c). Moreover, fatigue striations were observed in grains detachment sites (Fig. 5d). Generally, surface of the ZnAl4 alloy underwent a fast erosion - number of grains were detached, what leads to an uniform wearing upon further tests. b)

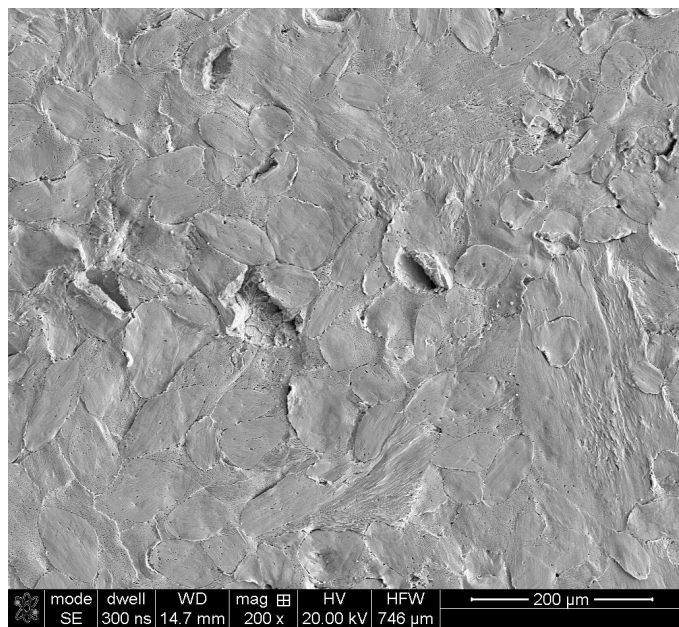

d)

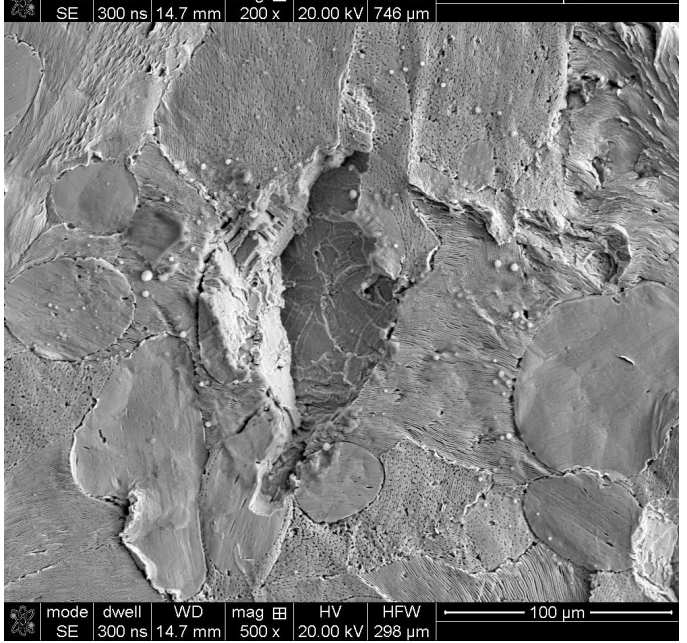

Fig. 5. SEM microphotographs showing surface changes in the ZnAl4 alloy upon the cavitation test carried out on the vibration stand a) depletion of $\eta+\alpha$ eutectic in grain and phase boundaries zones, b), c) erosion of $\eta$ solid solution dendrites and $\eta+\alpha$, d) fatigue striations 
The investigated ZnAl4 alloy exhibited different behavior upon testing on the jet impact stand. After 30 minutes of the exposition clearly visible signs of the material loss (in the form of radially oriented elongated grooves) were observed on the sample's surface (Fig. 6a). The cavitational destruction started with the erosion of $\eta+\alpha$ eutectic microregions and grains

a)

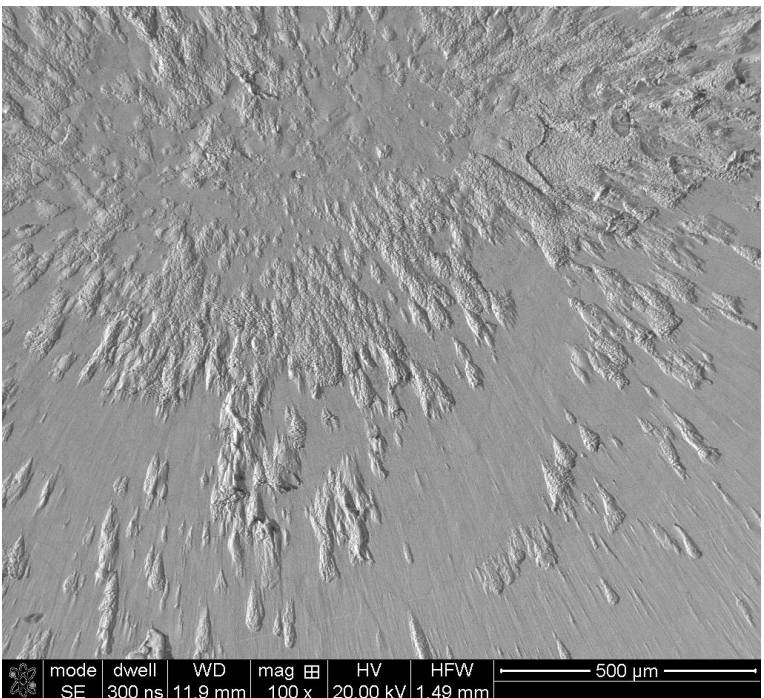

c)

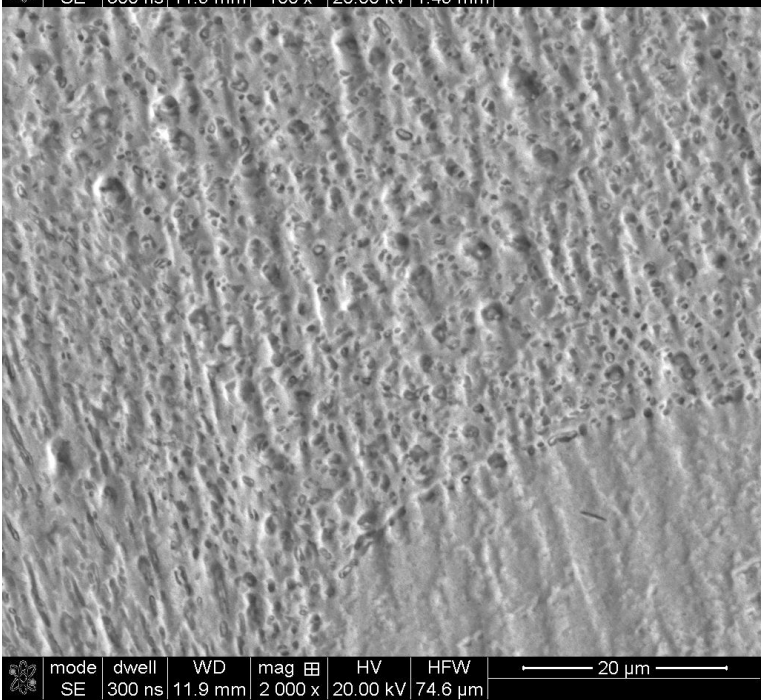

d)

of the $\eta$ solid solution. In these sites microgrooves originated from an explosion of single bubbles were formed (Fig. 6b-c). A slow erosion of $\eta+\alpha$ eutectic led to a damage of the $\eta$ solid solution (Fig. 6d). Further exposure resulted in an increase of eroded surface area, as well as deepening of grooves already existed in the material.

b)

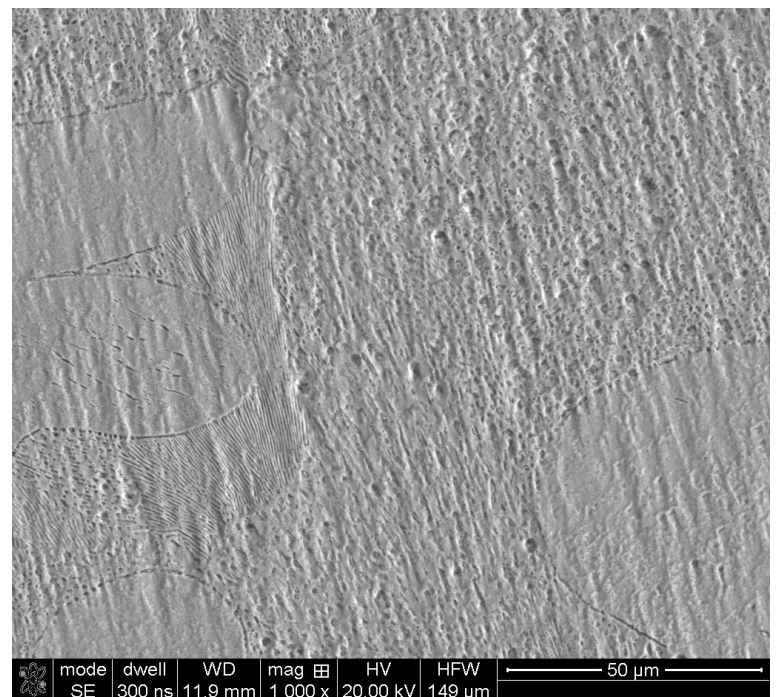

(1)

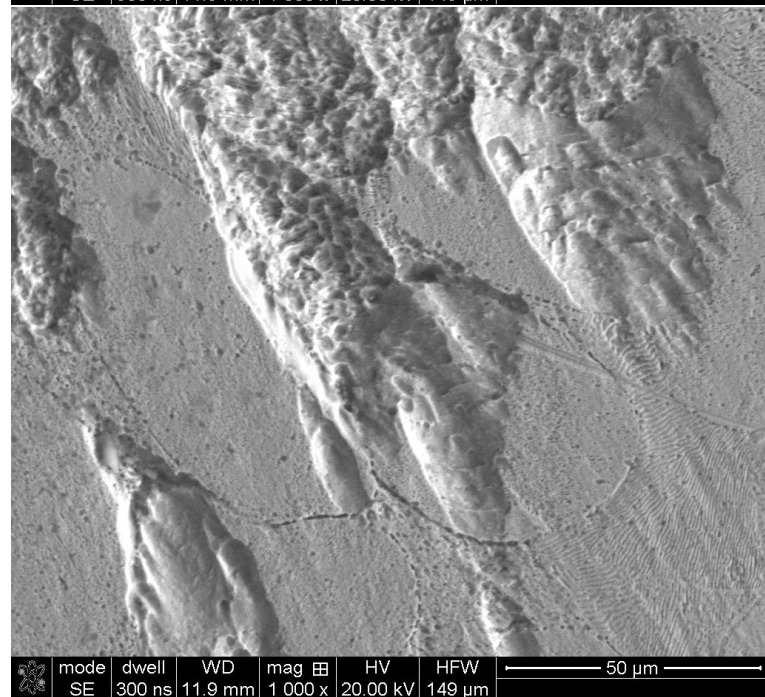

Fig. 6. SEM microphotographs showing surface changes in the ZnAl4 alloy upon the cavitation test carried out on the jet impact stand a) radially oriented elongated grooves; b), c) microgrooves in the $\eta+\alpha$ eutectic regions;

d) an erosion of the $\eta+\alpha$ eutectic and grain of the $\eta$ solid solution

A strike different mechanism of the cavitation erosion was recognized during tests carried out on the flow stand. In the initial stage of the cavitation wearing, softer $\eta$ solid solution was removed (Fig. 7a). This selective erosion leads to revealing of undamaged $\eta+\alpha$ eutectic areas (Fig. 7b), as well as to a further removing of the $\eta$ phase (Fig. 7c). The $\eta+\alpha$ eutectic exposed on cavitation impulses underwent crushing and a very slow erosion. 
a)

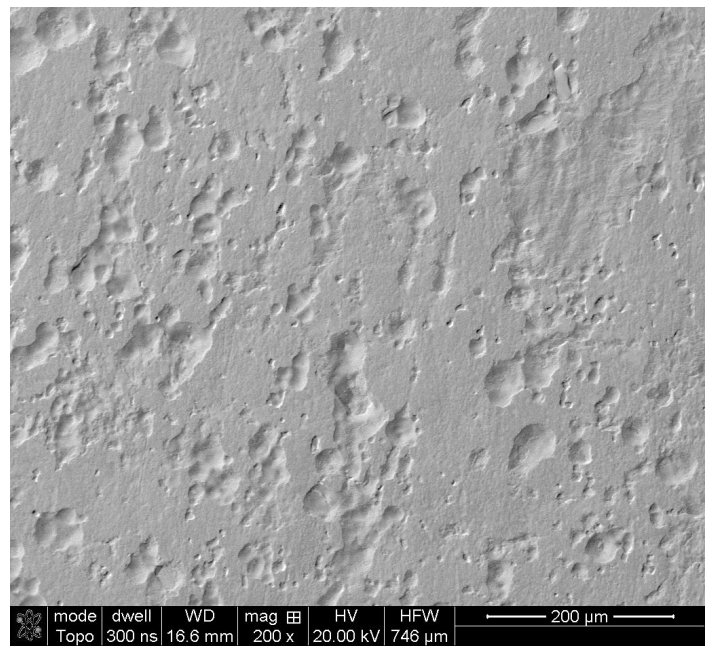

b)

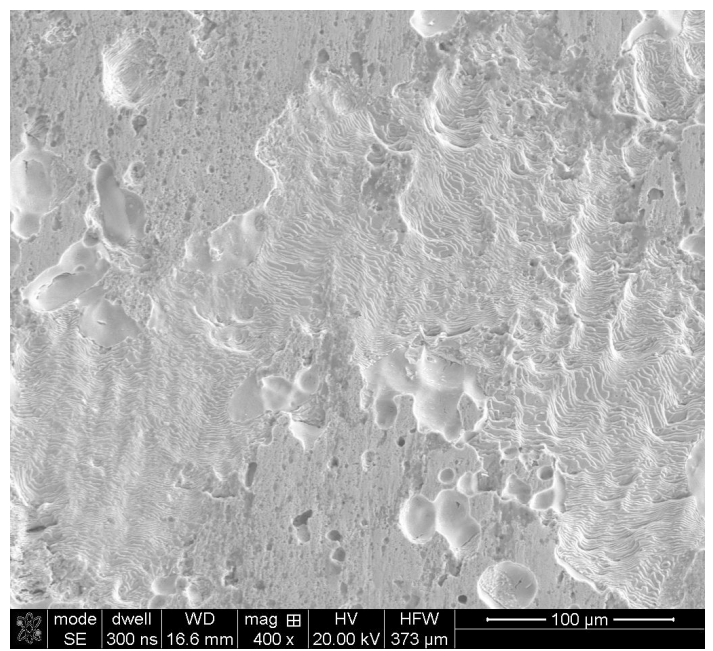

c)

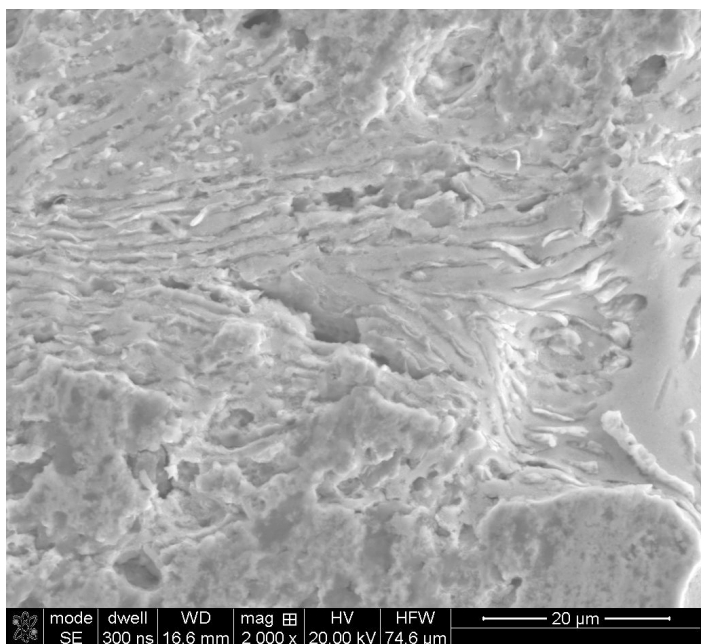

Fig. 7. SEM microphotographs showing surface changes in the ZnAl4 alloy upon the cavitation test carried out on the flow stand a), b) removing of the $\eta$ solid solution and revealing the $\eta+\alpha$ eutectic, c) a washout of the $\eta$ phase from the $\eta+\alpha$ eutectic
Mass change vs time curves of the destruction process upon testing on the considered laboratory stands are shown in Fig. 8.

Obtained results point toward that the course of cavitational destruction in the investigated material is strongly affected by the way of excitation of the cavitation process. The slowest kinetic of this destruction was recorded for the ZnAl4 alloy upon testing on the flow stand.

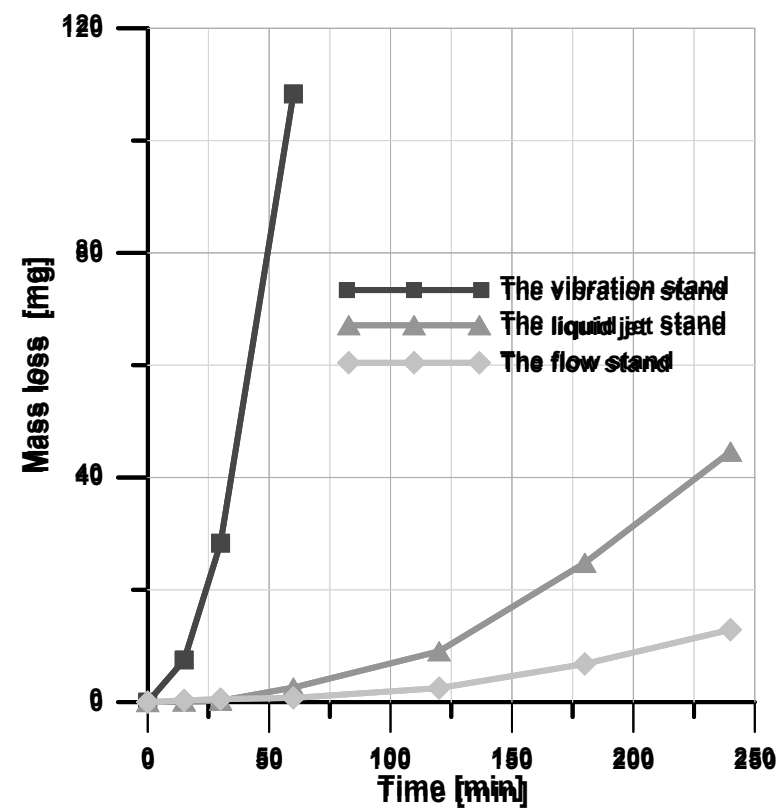

Fig. 8. The mass change vs. time curves for the investigated ZnAl4 as cast alloy

\section{Conclusion}

Results of conducted evaluation of the cavitational damage in the $\mathrm{ZnAl4}$ alloy carried out on three laboratory stands (vibration, jet impact and flow stands) point toward an existence of various destruction mechanisms. The cavitation erosion on vibration stand was initiated by the surface plastic deformation and single mass losses (both in the $\eta+\alpha$ eutectic and the $\eta$ solid solution areas). On the other hand, the cavitation process tested on the jet impact stand is started with the erosion of $\eta+\alpha$ eutectic followed by a damage of the solid solution. Finally, the cavitation on the jest impact stand begins with the washout of the $\eta$ solid solution and then the erosion of $\eta+\alpha$ eutectic. The above destruction mechanisms differ from the observed on the surface of the samples the same alloy which has been examined as a liquid impact stand [11].

A fatigue character of the process, which is typically found during cavitation erosion of metal materials, was observed only in ZnAl4 samples evaluated on the vibration stand. 


\section{Acknowledgements}

Scientific work funded by the Ministry of Education and Science in the years $2011 \div 2014$ as a research project No. N N507 231040.

\section{References}

[1] Brennen, C.E. (1995). Cavitation and Bubble Dynamics, Oxford University Press.

[2] Briggs, L.J. (1970). The Limiting Negative Pressure of Water, Journal of Applied Physics, Vol. 21, 721-722.

[3] Trevena, D.H. (1987). Cavitation and tension in liquids, IOP Publishing Ltd.

[4] Plesset, M.S. \& Chapman, R.B. (1971). Collapse of an Initially Spherical Vapour Cavity in the Neighborhood of a Solid Boundary, Jour. Fluid Mech., Vol. 47, Part 2, 283-290.
[5] Hickling, R. \& Plesset, M.S. (1963). Collapse and rebound of a spherical bubble in water, Phys. Fluids, No 7, 7-14.

[6] Naude, C.F. Ellis, A.T. (1961). On the mechanism of cavitation damage by non-hemispherical cavities collapsing in contact with a solid boundary, Journal of Basic Engineering, No. 83, 648-656.

[7] Garcia, R. \& Hammitt, F.G. (1967). Cavitation damage and correlations with material and fluid properties. Trans. ASME, Journal of Basic Engineering, No 89, 755-763.

[8] Gouesbet, G., Berlemont, A. (1993). Instabilities in Multiphase Flows. Plenum Press.

[9] Hammitt, F.G. (1980). Cavitation and Multiphase Flow Phenomena. McGraw-Hill.

[10] Hammitt, F.G. (1969). Impact and cavitation erosion and material mechanical properties. University of Michigan.

[11] Jasionowski, R. Zasada, D. \& Przetakiewicz, W. (2014). Cavitation Erosion Resistance Of Alloys Used In Cathodic Protection Of Hulls Of Ships, Archives of Metallurgy and Materials, Vol. 59 241-245. 